\title{
A Detailed View on the Proanthocyanidins in Ginkgo Extract EGb 761
}

\section{(우)(1) (요 $\ominus$}

Authors

Žarko Kulić ${ }^{*}$, Thomas Ritter ${ }^{2}$, Birgit Röck ${ }^{1}$, Jens Elsäßer ${ }^{1}$, Heike Schneider ${ }^{1}$, Stefan Germer ${ }^{2 *}$

Affiliations

1 Department of Preclinical Research and Development, Dr. Willmar Schwabe GmbH \& Co. KG, Karlsruhe, Germany

2 Department of Analytical Development, Dr. Willmar Schwabe GmbH \& Co. KG, Karlsruhe, Germany

Key words

EGb 761, quality, Ginkgo biloba, Ginkgoaceae, proanthocyanidins, HPLC

received accepted after revision published online

Bibliography

Planta Med 2022; 88: 398-404

DOI $\quad 10.1055 / a-1379-4553$

ISSN 0032-0943

(c) 2021. The Author(s).

This is an open access article published by Thieme under the terms of the Creative Commons Attribution-NonDerivative-NonCommercial-License, permitting copying and reproduction so long as the original work is given appropriate credit. Contents may not be used for commercial purposes, or adapted, remixed, transformed or built upon. (https://creativecommons.org/licenses/by-nc-nd/4.0/)

Georg Thieme Verlag KG, Rüdigerstraße 14,

70469 Stuttgart, Germany

Correspondence

Dr. Stefan Germer

Department of Analytical Development,

Dr. Willmar Schwabe GmbH \& Co. KG

Willmar-Schwabe-Straße 4, 76227 Karlsruhe, Germany

Phone: + 497214005445 , Fax: + 4972140058445

stefan.germer@schwabe.de

\section{ABSTRACT}

The Ginkgo extract EGb $761^{\circledR}$ manufactured with leaves of Ginkgo biloba has been continuously produced over decades at a large scale and is used as a clinically proven remedy for, among other things, the improvement of age-associated cognitive impairment and quality of life in patients with mild dementia. It belongs to the class of extracts addressed as quantified extracts according to the European Pharmacopeia. Accordingly, several compounds (e.g., flavone glycosides and terpene trilactones) are acknowledged to contribute to its clinical efficacy. Covering only about $30 \%$ of the mass balance, these characterized compounds are accompanied by a larger fraction of additional compounds, which might also contribute to the clinical efficacy and safety of the extract. As part of our systematic research to fully characterize the constituents of Ginkgo extract EGb 761, we focus on the structural class of proanthocyanidins in the present study. Structural insights into the proanthocyanidins present in EGb 761 and a quantitative method for their determination using HPLC are shown. The proanthocyanidins were found to be of oligomeric to polymeric structure, which yield delphinidin and cyanidin as main building blocks after acidic hydrolysis. A validated HPLC method for quantification of the anthocyanidins was developed in which delphinidin and cyanidin were detected after hydrolysis of the proanthocyanidins. The content of proanthocyanidins in Ginkgo extract EGb 761 was found to be approximately $7 \%$.

\section{ABBREVIATIONS \\ PACs proanthocyanidins \\ Ph. Eur. European Pharmacopeia \\ VIS visual light detection}

\section{Introduction}

The special Ginkgo extract EGb 761 [1] manufactured with leaves of Ginkgo biloba L. (Ginkgoaceae) is widely used for the improve- ment of age-associated cognitive impairment and quality of life in mild dementia, with proven clinical efficacy [2-4]. The Committee on Herbal Medicinal Products of the European Medicines Agency has published a monograph on G. biloba folium [5]. EGb 761 is registered as a drug in many countries worldwide. The herbal drug "Ginkgo leaf" is described in the monograph of the European Pharmacopeia [6] together with the monograph, Ginkgo Dry Extract, Refined and Quantified [7]. In accordance with the

* These authors contributed equally to this work. 
monograph, Herbal Drug Extracts, of the European Pharmacopeia [8], the classification as a quantified extract considers the fact that the specified active markers are likely to contribute to the clinical efficacy. However, in contrast to constituents with known therapeutic activity in standardized extracts, these active markers in quantified extracts cannot explain the clinical efficacy on their own [9]. In the case of the cited monograph of the European Pharmacopeia for quantified Ginkgo extract [7], the active markers are flavone glycosides, specified at 22.0 to $27.0 \%$, and terpene trilactones, specified at 2.6 to $3.2 \%$ for bilobalide and 2.8 to $3.4 \%$ for ginkgolides A, B, and C (calculated on the dried extract). In sum, these compounds yield approximately $30 \%$ of the dry extract. As part of our systematic research on constituents of the extract EGb 761 from leaves of $G$. biloba, we are working on elucidation of compounds not covered by the monograph. In this study, we focus on the class of structures usually addressed as PACs.

PACs are complex oligomeric and polymeric compounds in plants [10]. Depending on their degree of polymerization and the occurrence of different building blocks, fractions containing PACs show a multitude of compounds. Quantitative analytical approaches mainly applied classical methods such as color reactions and hydrolysis by hydrochloric acid followed by photometric assays [11] or used more modern methods like the (dimethylamino)cinnamaldehyde (DMAC) assay [12]. However, due to the manifold combination of the building blocks, linkage-types, stereochemical configuration, and polymerization degree, PACs are a highly challenging compound class that often require tailored methods for characterization [13]. The PACs in Ginkgo leaves were extensively studied by Qa'dan et al. [14] and were found to consist of B-type linkages $(4 \beta \rightarrow 6$ or $4 \beta \rightarrow 8)$ of mainly (epi-)gallocatechins and some minor amount of (epi-)catechins. However, structures and quantity of PACs in Gingko extracts used for finished products like EGb 761 were not studied in detail.

In the present study, an authentic fraction of PACs in Ginkgo extract EGb 761 was purified and subjected to a phytochemical characterization to establish a robust quantification method for PACs in Ginkgo extract EGb 761 through liquid chromatography and photometric detection that could be applied for routine analysis. In the native fraction of PACs in Ginkgo extract EGb 761, the multitude of compounds does not allow a good peak separation. Thus, we decided to use an approach where PACs are hydrolyzed and quantification is done using the resulting peaks of defined anthocyanidins. As anthocyanidins are known to exhibit low stability as reference substances, we used procyanidin B2 as a reference substance for calibration purposes. This compound can be handled easily and is known to be sufficiently stable. Additionally, this compound is commercially available. For calibration purposes, reference solutions were prepared by hydrolysis of procyanidin B2, and the resulting peak of cyanidin was used for quantitation. Sample solutions of Ginkgo extract EGb 761 were treated accordingly, and resulting peaks of major anthocyanidins delphinidin and cyanidin were used. The method was appropriately validated for precision, intermediate precision, linearity, accuracy, selectivity, and robustness and is suitable for the intended application. Besides the analysis of 11 batches of Ginkgo extract EGb 761 for which the content of PACs was to be determined, we also analyzed the purified authentic fraction of PACs from Ginkgo extract EGb 761. Due to the phyto- chemical complexity of this authentic PACs fraction, we derived a response factor for recalculation of the content of PACs in this fraction and further calculation of PACs in Ginkgo extract EGb 761.

\section{Results}

For molecular characterization and as the quantitation reference standard, a representative fraction of the PACs was purified by repeated column chromatography. The purified fraction was characterized by ${ }^{1} \mathrm{H}$-NMR, and the distribution of the molecular weight was determined by analytical gel permeation chromatography and HRMS ( $\triangleright$ Fig. 1). The NMR spectrum featured broad signals that are characteristic for polymers in general and PACs in particular ( $\triangleright$ Fig. 1 a). Besides a considerable amount of water observed as a characteristic resonance at 3.3 ppm in DMSO- $\mathrm{d}_{6}$, only minor sharp peaks of small-molecule impurities were observable, which were negligible in comparison to the integrals of the broad polymer signals. The water content was determined to be $9.19 \%$ by Karl Fischer titration using common standard procedures. In gel permeation chromatography, a large fraction of the proanthocyanidins appeared in the void volume, indicating a high polymerization degree. However, a considerable fraction appeared as a broad peak with a retention volume, which would correspond to tetra- to about hexameric procyanidins ( $\bullet$ Fig. $\mathbf{1}$ b), referred to as oligomers in the following. The molecular weights of these oligomers could be observed up to pentamers in HRMS spectra ( $\bullet$ Fig. 1 c). The observed mass patterns correspond to pure prodelphinidins or prodelphinidins with 1 procyanidin building block (mass difference of $16 \mathrm{amu}$ ), all with mainly B-type linkages and with A-type linkages only observed for dimers. Due to the mass range limitation of the Orbitrap detector and low signal-to-noise ratio, higher polymers could not be detected. However, the detected oligomers were not stable in solution. This was observed in gel permeation chromatography, where the oligomer peak disappeared after storage times of $>24 \mathrm{~h}$ in solution, yielding only polymers eluting in the void volume ( $\bullet$ Fig. $1 \mathbf{d}$ ). This behavior indicates that the PACs undergo a transformation in solution, which might be oxygen-induced polymerization or noncovalent aggregation. Other tested protocols for characterization of the PACs such as Diol-phase separation [15] did not yield PAC clusters as distinct peaks as expected from literature data. This observation may be due to the nature of the Ginkgo PACs, which seem to be prone to polymerization in solution and are mainly composed of prodelphinidins instead of procyanidins, the latter being the class of compounds applied to Diol-phase separation in literature [15]. Due to the negligible impurity signals in NMR spectra and a chromatogram that is characteristic for oligomers and polymers with no absorption for retention times of small molecules, the purified PACs were set to a content of $100 \%$ HPLC purity minus the water content of $9.19 \%$.

In order to confirm the flavan-3-ol monomer composition expected from the above HRMS data, the proanthocyanidin fraction was hydrolyzed with hydrochloric acid and analyzed by HPLC. For the characterization of the phenolic hydrolysis products, a gradient HPLC method was used, with a detection wavelength specific for anthocyanidins ( $\bullet$ Fig. 2). Since anthocyanidins are the only compounds in the Ginkgo extract that exhibit absorption at $530 \mathrm{~nm}$ after hydrolysis, the method for quantification not only 


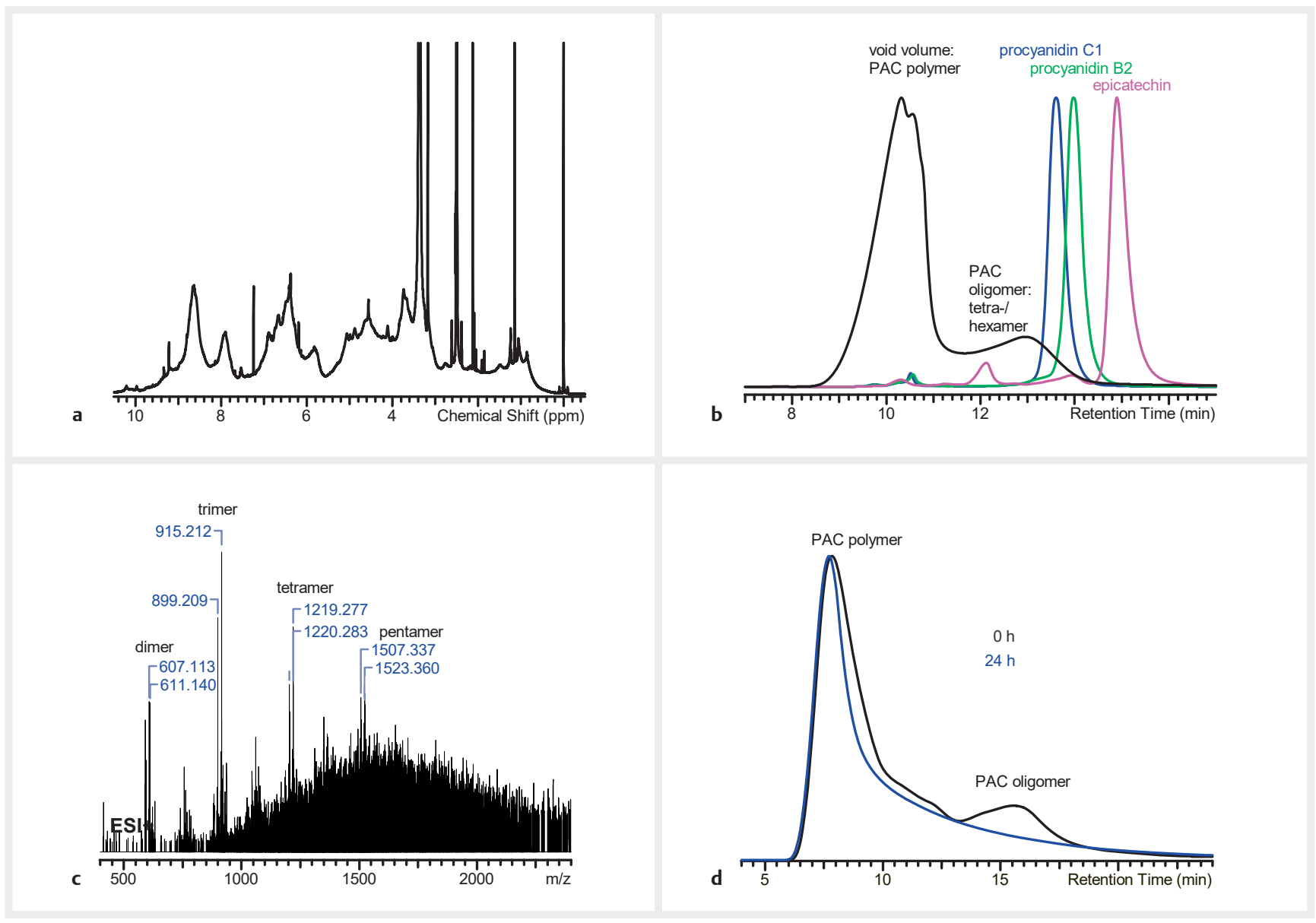

- Fig. 1 a ${ }^{1} \mathrm{H}$-NMR spectrum of polymeric proanthocyanidins (PACs) purified from EGb 761. The broad signals are characteristic for polymers and comprise the major intensity of the spectrum. Sharp signals of small molecule impurities have low intensity compared to the polymer signals, and thus, can be considered as negligible. Only the water resonance at $3.3 \mathrm{ppm}$ is of considerable intensity. $\mathbf{b}$ Gel permeation chromatograms of PACs (black), and proanthocyanidins of different size for calibration: procyanidin C1/trimer (blue), procyanidin B2/dimer (green), and epicatechinmonomer (pink) detected optically at a wavelength of $230 \mathrm{~nm}$. A large fraction of the PACs elutes in the void volume at the retention time of 9-11 min. A minor fraction elutes at the retention time of 12-13 min, indicating a tetra- to hexameric state of the proanthocyanidins. In the PAC fraction, no absorption at $230 \mathrm{~nm}$ is observable for molecules smaller than procyanidin B2, indicating that the fraction consists mainly of PAC oligomers and polymers. c ESI+HRMS Spectrum of PACs. Mass patterns of dimers to pentamers are observable for prodelphinidins or prodelphinidins with 1 cyanidin monomer due to the mass difference of 1 oxygen atom (16 amu). The detected masses are characteristic for B-type linkage, with masses for A-type linkage only observable for the dimer (607 amu). d Gel permeation chromatograms of PACs detected at a wavelength of $230 \mathrm{~nm}$. Measurement directly after dissolving the sample (black), and after $24 \mathrm{~h}$ in solution (blue). The oligomer fraction depletes over time in the solution, resulting in elution only in the void volume. This behavior indicates a polymerization of the proanthocyanidins in solution. The different retention times are due to 2 different columns used: MCX analytical $100 \AA ̊$ column (b) and PolySep-SEC GFC-P3000 column (d).

can be used for the purified fraction but also the whole extract. The detected anthocyanidins were delphinidin, cyanidin, pelargonidin, and an anthocyanidin compound that could not yet be identified, in the relative integral-ratio of $77.7 \%, 15.9 \%, 3.0 \%$, and $3.4 \%$, respectively. The hydrolysis compounds were identified by spiking with reference compounds. Although the anthocyanidins are in a charged state, the compounds could not be detected by ESI mass spectrometry. In this respect, the unknown hydrolysis compound could not be identified by a naïve approach. Spiking was tried with luteolinidin, fisetinidin, peonidin, petunidin, and malvidin, which, however, did not exhibit the retention time of the unknown peak (data not shown). The monomer composition observed after hydrolysis was consistent with the composition determined by HRMS, with (epi-)gallocatechin (or delphinidin after hydrolysis) being the major monomer with a minor content of (epi-)catechin (or cyanidin after hydrolysis). This observation is also consistent with literature data for Ginkgo PACs [14].

In summary, the EGb 761 PACs consist of polymers and dimers to about hexamers. The latter polymerize or aggregate in solution to a high polymeric form over time. The polymeric proanthocyanidins form mainly delphinidin and cyanidin, trace amounts of pelargonidin, and one unknown anthocyanidin compound as hydrolysis products.

Several HPLC methods were evaluated for quantitation of PACs in Ginkgo Extract EGb 761. In the monograph Ginkgo Dry Extract, Refined and Quantified by the European Pharmacopeia [7], a method is described for flavonoids after acidic hydrolysis of Ginkgo flavone glycosides using HPLC and photometric detection. During 
our research, we observed that when using this original method of the European Pharmacopeia, a characteristic peak pattern was detectable at a wavelength of $530 \mathrm{~nm}$ within the first few min. The main emerging peaks were found to be the anthocyanidins delphinidin and cyanidin beside two smaller peaks at retention times of 5.8 and $6.3 \mathrm{~min}$. As the original method focuses on quantitation of flavonoids, we used the method for additional quantitation of anthocyanidins within another retention timeframe and detection wavelength. For this reason, we adapted the method regarding the gradient elution to shorten the run time.

The purified PAC fraction as described above was subjected to the HPLC assay after hydrolysis. As the HPLC assay of the fraction is essential for the calculation of the content, this assay was done in replicates $(n=6)$ with completely independent sample preparations and was repeated with new samples $(n=6)$ after $1 \mathrm{wk}$. The result was the content for PACs of $43.4 \%$ (day 1 ) and $42.2 \%$ (day 2 ) in this fraction, calculated as procyanidin $\mathrm{B} 2$. As result, the mean value of $42.8 \%$ was taken, corresponding to $47.1 \%$ calculated on the waterfree fraction considering the water content of $9.19 \%$. The emerging peaks of delphinidin and cyanidin in this fraction were quantified as cyanidin, and the content of PACs in this fraction was calculated using a calibration of the emerging peak of cyanidin in the standard solutions of procyanidin B2 as described. Since the PAC fraction purified from Ginkgo Extract EGb 761 was set to $100 \%$ by convention, a conversion factor was needed for determining the content using procyanidin B2. The applied HPLC assay correlates to a response factor of 2.12 for the water-free fraction. To receive the corresponding content of PACs in Ginkgo extract EGb 761, this response factor must be considered. This means that the results of anthocyanidins for the extract must be multiplied by 2.12 to get the content of genuine EGb 761 PACs. In this context, 11 batches of Ginkgo extract EGb 761 were subjected to the HPLC assay, and the contents of PACs determined as described above are summarized in $\downarrow$ Table 1 . Thus, the mean content of PACs in the batches of Ginkgo extract EGb 761 was $6.98 \%$, with a range from $6.02 \%$ to $7.36 \%$.

\section{Discussion}

The polymeric PACs present in G. biloba extract EGb 761 exhibit an uncommon characteristic composition of hydrolysis products with the major compounds being delphinidin and cyanidin. The extent of polymerization seems to include an oligomeric fraction of mainly B-type dimers to hexamers that were, however, unstable in solution and form higher polymers over time. Since the polymerization takes place in solution, it is possible that the major polymeric fraction is not the genuine state of the PACs but rather a reaction product happening during extraction and isolation. PACs derived from hawthorn, cranberries, or grape seed are mainly composed of (epi-)catechin building blocks (or cyanidin after hydrolysis). In contrast, the PACs present in Ginkgo extract have (epi-)gallocatechin as the major building block (or delphinidin after hydrolysis). This difference may be responsible for the fact that some methods for characterization of PACs reported in literature, like Diol-phase separation, do not lead to usable results in the case of Ginkgo PACs.

Due to the complexity of this fraction with polymeric structures, the objective of this study was to provide a new method for easy and robust quantitation of PACs in Ginkgo extract EGb

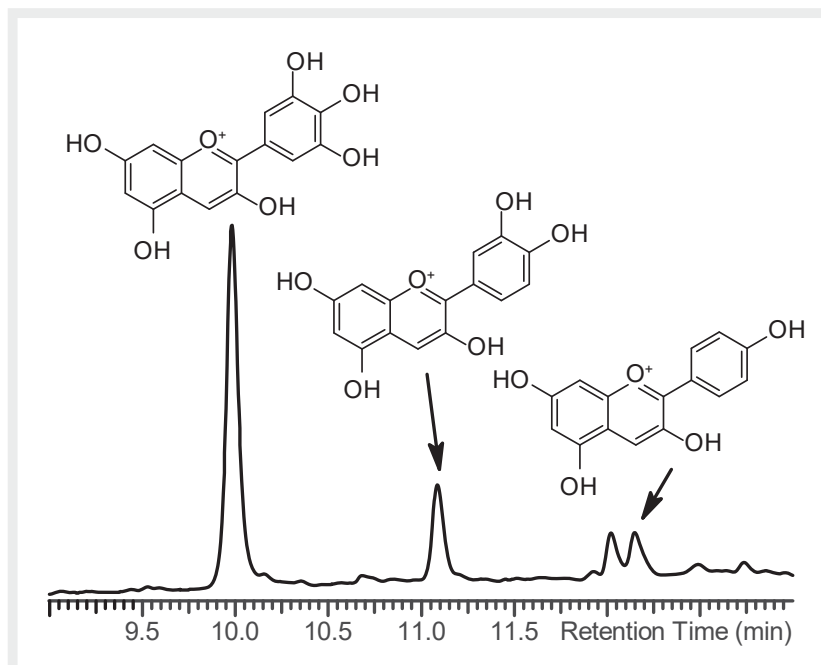

- Fig. 2 HPLC chromatogram of the polymeric proanthocyanidins (PACs) purified from EGb 761 after acidic hydrolysis detected at $530 \mathrm{~nm}$. The major hydrolysis product is delphinidin eluting at $10.0 \mathrm{~min}$, with some minor fraction of cyanidin (eluting at $11.1 \mathrm{~min}$ ) and pelargonidin (eluting at $12.2 \mathrm{~min}$ ). The elution peak at $12.0 \mathrm{~min}$ could not yet be characterized.

- Table 1 Results for PACs in 11 batches of Ginkgo extract EGb 761. Results calculated as procyanidin B2 are transferred to the authentic PACs fraction by multiplication with the conversion factor 2.12 .

\begin{tabular}{|c|l|l|}
\hline \begin{tabular}{|} 
Batch Ginkgo \\
Extract EGb 761
\end{tabular} & $\begin{array}{l}\text { Assay PACs } \\
\text { calculated as } \\
\text { procyanidin B2 }\end{array}$ & $\begin{array}{l}\text { Assay PACs calcu- } \\
\text { lated as authentic } \\
\text { PACs fraction }\end{array}$ \\
\hline 1 & $3.46 \%$ & $7.34 \%$ \\
\hline 2 & $3.44 \%$ & $7.29 \%$ \\
\hline 3 & $3.38 \%$ & $7.17 \%$ \\
\hline 4 & $3.35 \%$ & $7.10 \%$ \\
\hline 5 & $3.47 \%$ & $7.36 \%$ \\
\hline 6 & $3.06 \%$ & $6.49 \%$ \\
\hline 7 & $2.84 \%$ & $6.02 \%$ \\
\hline 8 & $3.15 \%$ & $6.68 \%$ \\
\hline 9 & $3.45 \%$ & $7.31 \%$ \\
\hline 10 & $3.32 \%$ & $7.04 \%$ \\
\hline 11 & $3.30 \%$ & $7.00 \%$ \\
\hline Standard deviation & $0.20 \%$ & $6.98 \%$ \\
\hline & & \\
\hline
\end{tabular}

761 by HPLC and photometric detection. The method developed in this study is based on the pharmacopeial method for flavonoids in the monograph Ginkgo Dry Extract, Refined and Quantified from the European Pharmacopeia [7], which we adopted to quantify PACs. Instead of delphinidin or cyanidin, procyanidin B2 is suggested as a standard substance since it shows sufficient stability 
for handling at room temperature and is easily commercially available. The analytes delphinidin and cyanidin are formed in situ during the hydrolysis of both standard solution and sample solutions and are analyzed together within the same chromatographic run within appropriate run times. A product-specific correlation of the results was derived by analysis of the authentic fraction of EGb 761-PACs. The new method is highly selective and supports the obtained results by additional information concerning the building blocks of PACs in Ginkgo. As a chromatographic method, automation is easily possible in contrast to classical methods based on photometry that require more manual steps.

Our data show that PACs were present in the batches tested with a mean quantity of approximately $7 \%$. Thus, PACs can now be considered to be a quantitatively relevant portion of natural compounds within the Ginkgo extract EGb 761. Due to the well-defined production process, the content of PACs in EGb 761 is maintained within a constant narrow range, including the batches tested in clinical trials for demonstration of clinical efficacy of EGb 761. The polymerization degree and building blocks of PACs are relatively well known, but the exact structures still require elucidation.

\section{Materials and Methods}

\section{Purification of PACs}

One $\mathrm{kg}$ of Ginkgo extract EGb 761 was dissolved in $2 \mathrm{~L}$ deionized water at $60^{\circ} \mathrm{C}$. After cooling the solution to room temperature $\left(25^{\circ} \mathrm{C}\right)$, the solution was filtered through a porous glass filter with a pore size of $10-16 \mu \mathrm{m}(\mathrm{G} 4)$ to remove a small amount of insoluble residue. The insoluble residue was suspended in about $100 \mathrm{~mL}$ deionized water, sonicated for $5 \mathrm{~min}$, and filtered again. The insoluble residue consists mainly of ginkgolides $(\sim 12 \mathrm{~g})$. The extract solution was applied on water-presoaked Sephadex LH-20 (GE Healthcare) material in a column, with a gel bed volume of $12.5 \mathrm{~L}$. The elution started with $30 \mathrm{~L}$ water to remove all hydrophilic compounds. The upper $4.5 \mathrm{~L}$ of the gel bed containing the PACs were removed, suspended in aqueous $25 \%$ vol. ethanol, and placed into a separate column. A serial elution followed with $14 \mathrm{~L}$ of aqueous $25 \%$ vol. ethanol, followed by $10 \mathrm{~L}$ of $96 \%$ vol. ethanol. These 2 fractions were discarded. The PACs were eluted with $8 \mathrm{~L}$ of an aqueous $70 \%$ vol. acetone solution. This protocol was repeated with another $1 \mathrm{~kg}$ of Ginkgo extract EGb 761. The PAC fractions from the first and second purification batch were combined, and the solvent was evaporated. For final purification, the solid residue $(78 \mathrm{~g}$ ) was dissolved in $0.7 \mathrm{~L}$ aqueous $50 \%$ vol. ethanol and applied once again on a fresh $\mathrm{LH}-20$ material presoaked with aqueous $50 \%$ vol. ethanol and a gel bed volume of $5.4 \mathrm{~L}$. The elution started with $5.9 \mathrm{~L}$ aqueous $50 \%$ vol. ethanol to remove remaining impurities. The PACs were eluted with $14.6 \mathrm{~L}$ of an aqueous $70 \%$ vol. acetone solution, yielding $69 \mathrm{~g}$ dry residue.

\section{Molecular characterization of PACs}

NMR spectra were acquired on a Bruker Avance III HD $600 \mathrm{MHz}$ system equipped with an inverse Prodigy $\mathrm{TCl}$ cryoprobe. We dissolved $24.5 \mathrm{mg}$ of PACs in $600 \mu \mathrm{L}$ DMSO- $\mathrm{d}_{6}$. 1D- ${ }^{1} \mathrm{H}$ NMR spectra were measured using tetramethylsilane as internal standard with 32 accumulated scans with a spectral width of 18 ppm, an offset of $8 \mathrm{ppm}$ for the transmitter frequency, and a digital resolution of $64 \mathrm{k}$ data points. The spectra were recorded and processed with Bruker Topspin software (v3.6pl7) and analyzed with ACD/Labs Spectrus Processor (v2017.2.1).

ESI-HRMS spectra were acquired on a Thermo Orbitrap Fusion mass detector. PACs were dissolved to a concentration of $1 \mathrm{mg} /$ $\mathrm{mL}$ in a mixture of acetonitrile-water-formic acid 49.75:49.75: $0.50(\mathrm{v} / \mathrm{v} / \mathrm{v})$ and injected with $5 \mu \mathrm{L} /$ min directly into the ESI source by a syringe pump. Spectra were measured in positive ion mode with an ionization voltage of $3500 \mathrm{~V}$, a high mass range of 400 $6000 \mathrm{~m} / \mathrm{z}$, and a resolution of $30 \mathrm{k}$. The spectra were recorded and processed by Thermo Xcalibur software (v4.3) and analyzed by ACD/Labs Spectrus Processor (v2017.2.1).

HPLC analysis of the molecular weight by gel permeation chromatography was carried out on a Hitachi LaChrom System, consisting of an autosampler, a high pressure mixing pump, a column oven, and a diode array detector coupled in-line with a refractive index detector. Two methods were used for analysis. One method used a Polymer Standards Service MCX analytical $100 \AA$ column $(5 \mu \mathrm{m}, 8 \times 300 \mathrm{~mm})$ with a total run time of $30 \mathrm{~min}$, and the other method used a Phenomenex PolySep-SEC GFC-P3000 column $(7.8 \times 300 \mathrm{~mm})$ with a total run time of $45 \mathrm{~min}$. Both methods applied an isocratic elution of an aqueous $40 \%$ vol. acetonitrile solution with a flow rate of $0.8 \mathrm{~mL} / \mathrm{min}$; column temperature: $50^{\circ} \mathrm{C}$; detection: UV at $230 \mathrm{~nm}$; injection volume: $10 \mu \mathrm{L}$; sample concentration: $4.5 \mathrm{mg} / \mathrm{mL}$ in elution solvent. For calibration of the retention times, epicatechin $0.8 \mathrm{mg} / \mathrm{mL}$ (monomer), procyanidin B2 $1.0 \mathrm{mg} / \mathrm{mL}$ (dimer), and procyanidin $\mathrm{C} 10.8 \mathrm{mg} / \mathrm{mL}$ (trimer) were used, respectively. Standards for higher oligomeric proanthocyanidins were not commercially available. The molecular weight of the sample was estimated by extrapolating the elution volumes of the mono-, di- and trimer.

Water content was determined by Karl Fischer titration using common standard procedures.

Qualitative HPLC analysis of the hydrolysis products was carried out on a Thermo UltiMate 3000 system with autosampler WPS-3000 TFC, pump HPG-3400 RS, detector DAD-3000 RS, and column oven TCC-3000 RS. The anthocyanidin hydrolysis products were analyzed using a Waters Cortecs UPLC C18 column $(1.6 \mu \mathrm{m}$, $2.1 \times 150 \mathrm{~mm}$ ); solvent system A: water/acetonitrile/formic acid 98.7: 1:0.3 (v/v/v), solvent system B: acetonitrile-water-formic acid 98.7: 1:0.3 (v/v/v); 15-50\% B over 17 min, 50-100\% B over 2 min following rinsing with $100 \%$ B for 2 min, $100-15 \%$ B for $1 \mathrm{~min}$ and equilibration with $15 \% \mathrm{~B}$ for $5 \mathrm{~min}$; flow rate: $0.2 \mathrm{~mL} /$ min; column temperature: $25^{\circ} \mathrm{C}$; detection: $530 \mathrm{~nm}$; injection volume: $5 \mu \mathrm{L}$; sample concentration: $5 \mathrm{mg} / \mathrm{mL}$ in elution solvent. For calibration of the retention times, analytical grade standards were used; delphinidin, cyanidin, pelargonidin, peonidin, and malvidin were purchased from Sigma Aldrich, luteolinidin and fisetinidin were obtained from Extrasynthese, and petunidin was purchased from Cayman Chemical.

The HPLC method that was used for quantification and verification of the composition is described below. 


\section{Quantification of PACs}

Test samples

Eleven batches of Ginkgo extract EGb 761 were used to analyze the quantity of PACs with the method described. Specimens are deposited in the archive of Dr. Willmar Schwabe GmbH \& Co. KG, Germany. The results were used for additional calculation with an extract-specific response factor for the purified authentic PACs fraction of Ginkgo extract EGb 761 (see below).

\section{Solvents, reagents, and chemicals}

Methanol (p.a.), o-phosphoric acid, and hydrochloric acid were purchased from Merck. The deionized water was obtained by a water purification system (Evoqua, Water Technologies). For the preparation of the hydrolysis solution, 1 part methanol and 1 part 1.5 $\mathrm{M}$ hydrochloric acid were mixed ( $\mathrm{v} / \mathrm{v})$.

\section{Reference standard}

Procyanidin B2 (CAS number 29 106-49-8) with purity $\geq 98 \%$ was purchased from Cayman Chemical Company. The reference standard procyanidin B2 was hydrolyzed in the reference solution to form the reference peak cyanidin in situ (see below).

\section{Preparation of standard solutions}

Reference standard (procyanidin B2) is weighed exactly into appropriate volumetric flasks and dissolved in the hydrolysis solution to obtain a standard solution with $100 \mu \mathrm{g}$ procyanidin B2/mL (example chromatogram, see $>$ Fig. $\mathbf{3 a}$ ). This standard solution was diluted with the hydrolysis solution to receive an additional standard solution with $10 \mu \mathrm{g}$ procyanidin B2/mL (example chromatogram, see $>$ Fig. $\mathbf{3 b}$ b). The standard solutions were hydrolyzed in parallel and in the same way as described for the sample solutions (see below). The standard solutions appeared clear after hydrolysis and were used directly for HPLC analysis without prior filtration.

\section{Preparation of sample solutions}

Samples of $100 \mathrm{mg}$ Ginkgo extract EGb 761 were weighed exactly into $25 \mathrm{~mL}$ volumetric flasks, filled up to volumes with hydrolysis solution, and dissolved in an ultrasonic bath (SONOREX Super RK510, 160/320 W, $35 \mathrm{kHz}$, Company Bandelin) for $10 \mathrm{~min}$ at room temperature. The obtained test solutions were hydrolyzed in a boiling water bath in tightly closed $10 \mathrm{~mL}$ hydrolysis tubes with screw caps remaining above the surface. We determined $45 \mathrm{~min}$ to be the optimal duration to yield complete hydrolysis. After hydrolysis, the closed tubes were cooled in an ice bath and stored at ambient conditions for tempering to room temperature $\left(23^{\circ} \mathrm{C}\right)$. The sample solutions appeared clear after hydrolysis and were used directly for HPLC analysis without prior filtration (example chromatogram, see $>$ Fig. 3 c).

\section{Analysis of PACs in samples of Ginkgo extract EGb 761 and EGb 761 PACs}

The hydrolyzed standard solutions and sample solutions of the purified PACs fraction and of the 11 batches of Ginkgo extract EGb 761 were transferred directly into individual vials without prior filtration and were submitted for HPLC analysis.
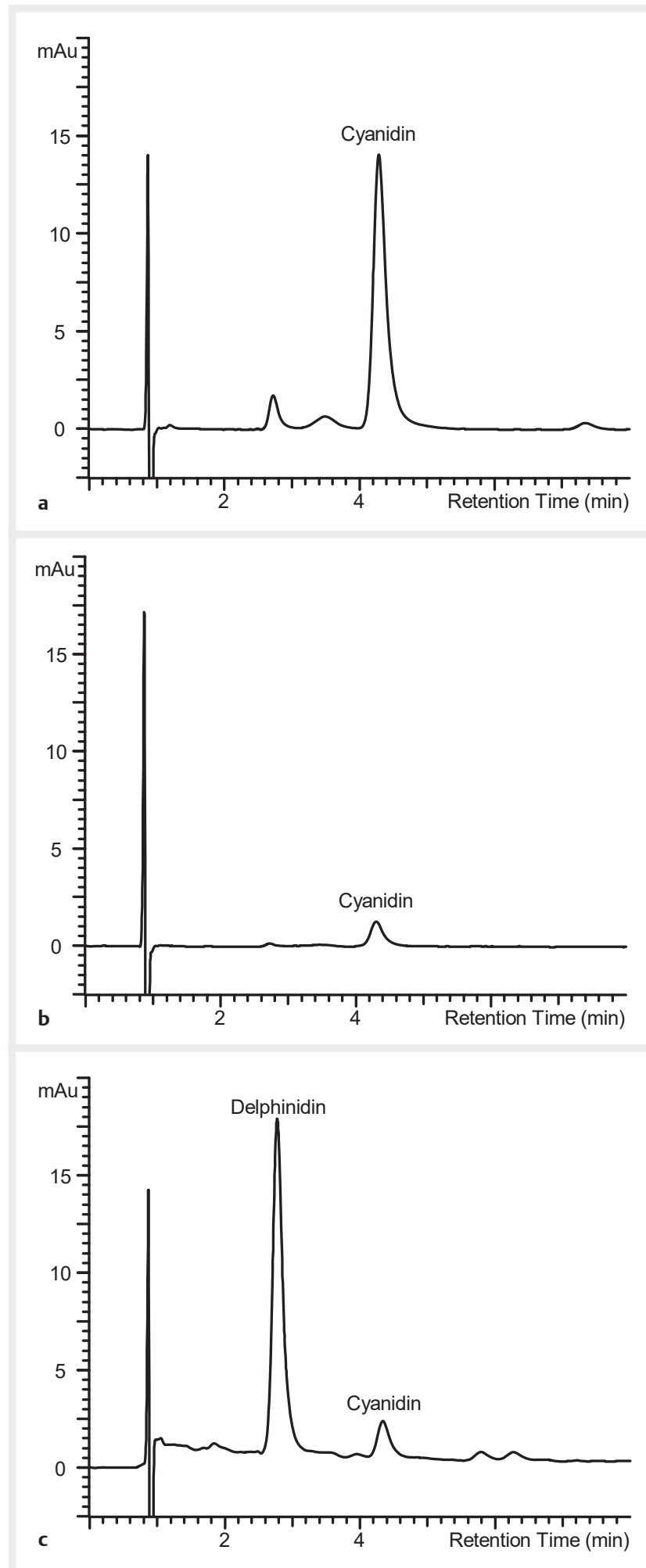

- Fig. 3 a HPLC chromatogram of the standard solution with approximately $100 \mu \mathrm{g}$ procyanidin B2/mL after hydrolysis, detected at $530 \mathrm{~nm}$. b HPLC chromatogram of the standard solution with approximately $10 \mu \mathrm{g}$ procyanidin $\mathrm{B} 2 / \mathrm{mL}$ after hydrolysis, detected at $530 \mathrm{~nm}$. c HPLC chromatogram of one sample solution after hydrolysis, detected at $530 \mathrm{~nm}$. The retention times differ from the method used for molecular characterization ( $\vee$ Fig. 2). The integrals of the measured peaks are within the range of the calibration. 


\section{Chromatography}

HPLC was performed on a Thermo UltiMate 3000 system with an autosampler WPS-3000 TRS, a pump LPG-3400 RS, a detector MWD-3000 RS, and a column oven TCC-3000 SD using a Kromasil C18 (5 $\mu \mathrm{m}, 4 \times 125 \mathrm{~mm}$ ) column (MZ-Analysentechnik) without pre-column. The mobile phase consisted of water adjusted to $\mathrm{pH} 2.0$ with o-phosphoric acid $85 \%$ solution (phase $\mathrm{A}$ ) and methanol (phase B). The following gradient was applied at a flow rate of $1.0 \mathrm{~mL} / \mathrm{min}$ : isocratic from $0.00-1.00 \mathrm{~min}$ at $60 \%$ Eluent $A$, from 1.00-8.00 min linear from $60 \%$ Eluent $A$ to $54.5 \%$ Eluent $A$ following 8.00-9.00 min linear 0\% Eluent A, 4 min column wash with $0 \%$ Eluent $A$, from $13.00-13.50 \mathrm{~min}$ to $60 \%$ Eluent $A$ and $6.5 \mathrm{~min}$ equilibration period with $60 \%$ Eluent $A$, resulting in a total run time of $20.00 \mathrm{~min}$. VIS detection wavelength of $530 \mathrm{~nm}$, a column temperature of $25^{\circ} \mathrm{C}$, and an injection volume of $10 \mu \mathrm{L}$ were applied.

The retention time for delphinidin was approximately $2.8 \mathrm{~min}$, for cyanidin $4.3 \mathrm{~min}$, and for pelargonidin $6.3 \mathrm{~min}$, respectively. Quantitation was done for the peaks of delphinidin and cyanidin individually using the standard solutions of hydrolyzed procyanidin B2, and both results were added for further calculation. Delphinidin was calculated as cyanidin. Smaller peaks like pelargonidin were not considered in the sample solutions for quantitation, due to being off of the dynamic detection range.

\section{Data analysis}

Data processing and analysis were carried out using Chromeleon 7.2 SR5 software (Thermo).

\section{Validation data}

The method was comprehensively validated addressing the parameters precision, intermediate precision, linearity, accuracy, selectivity, and robustness. A test for precision with $n=6$ individual sample preparations of Ginkgo extract EGb 761 showed a relative standard deviation of $6.46 \%$. The test was repeated after 1 week for evaluation of intermediate precision with new sample preparation and new calibration and showed a relative standard deviation of $5.61 \%$. Linearity was tested by linear regression with 12 concentrations between $0.602 \mu \mathrm{g}$ procyanidin B2/mL to $120.400 \mu \mathrm{g}$ procyanidin B2/mL. Correlation coefficient was 0.999946 with $y$-intercept -0.0181 area and slope 0.0353 area $/ \mu \mathrm{g} / \mathrm{mL}$. As the reference substance cyanidin is formed in situ by acidic hydrolysis of procyanidin B2, accuracy was addressed by the addition of procyanidin B2 to an accurately weighed quantity of Ginkgo extract EGb 761. The quantity of Ginkgo extract EGb 761 was half as described in the test procedures and was supplemented by the addition of procyanidin B2 to a final quantity of $80 \%$ and $120 \%$ of the theoretical content of PACs in the sample of Ginkgo extract EGb 761. The test was done twice with recovery rates for cyanidin of $97.3 \%$ and $95.7 \%$ for the $80 \%$ concentration level and $95.9 \%$ and $95.7 \%$ for the $120 \%$ concentration level. Selectivity was shown with view on the peak identification of delphinidin, cyanidin, and pelargonidin and their good chromatographic separation. The robustness of the method was tested by variation of method parameters described in the test procedures like column temperature and $\mathrm{pH}$ value of the mobile phase $\mathrm{A}$ within relevant ranges and was considered to be suitable. Also, the time required for an analysis series was suitable to guarantee the stability of reference and sample solutions.

\section{Contributors' Statement}

Purification of the material: B. Röck; data collection: T. Ritter, J. Elsäßer, H. Schneider; design of the study: Z. Kulic, S. Germer; analysis and interpretation of the data: Z. Kulic, S. Germer; drafting the manuscript: Z. Kulic, S. Germer; these authors contributed equally: Z. Kulic, S. Germer.

\section{Acknowledgements}

We thank Andreas Butterer, Department of Preclinical Research and Development, Dr. Willmar Schwabe GmbH \& Co. KG, Karlsruhe, Germany, for the measurement of the NMR spectra.

\section{Conflict of Interest}

All authors are employees of Dr. Willmar Schwabe GmbH \& Co. KG, Germany.

\section{References}

[1] EGb $761^{\circledR}$ is a proprietary extract manufactured by Dr. Willmar Schwabe GmbH \& Co. KG

[2] Kasper S. Clinical data in early intervention. Int Psychogeriatr 2012; 24 (Suppl. 1): S41-S45

[3] Ihl R. Ginkgo biloba extract EGb $761^{\circledR}$ : clinical data in dementia. Int Psychogeriatr 2012; 24 (Suppl. 1): S35-S40

[4] Müller WE, Eckert A, Eckert GP, Fink H, Friedland K, Hörr R, Ihl R, Kasper S, Möller HJ. Ginkgo Spezialextrakt $761^{\circledR}\left(\right.$ Tebonin $\left.^{\circledR}\right)$. Psychopharmakotherap 2016; 3: 102-117

[5] EMA/HMPC/321097/2012, European Union herbal monograph on Ginkgo biloba L., folium

[6] Ph. Eur. 10.0, monograph 1828, Ginkgo Leaf

[7] Ph. Eur. 10.0, monograph 1827, Ginkgo Dry Extract, Refined and Quantified

[8] Ph. Eur. 10.0, monograph 0765, Herbal Drug Extracts

[9] Waimer F, Stumpf H. Quality of quantified plant extracts. DAZ2006; 17: 1842-1847

[10] Rauf A, Imran M, Abu-Izneid T, lahtisham-Ul-Haq, Patel S, Pan X, Naz S, Sanches Silva A, Saeed F, Rasul Suleria HA. Proanthocyanidins: A comprehensive review. Biomed Pharmacother 2019; 116: 108999

[11] Lang F, Wilhelm E. Quantitative determination of proanthocyanidins in Ginkgo biloba special extracts. Pharmazie 1996; 10: 734-737

[12] Birmingham AD, Esquivel-Alvarado D, Maranan M, Krueger CG, Reed JD. Inter-laboratory validation of 4-(dimethylamino) cinnamaldehyde (DMAC) assay using cranberry proanthocyanidin standard for quantification of soluble proanthocyanidins in cranberry foods and dietary supplements, first action official method SM: 2019.06. J AOAC Int 2020. doi:10.1093/jaoacint/qsaa084

[13] van Dooren I, Foubert K, Theunis M, Naessens T, Pieters L, Apers S. Advantages of a validated UPLC-MS/MS standard addition method for the quantification of A-type dimeric and trimeric proanthocyanidins in cranberry extracts in comparison with well-known quantification methods. J Pharm Biomed Anal 2018; 148: 32-41

[14] Qa'dan F, Mansoor K, AL-Adham I, Schmidt M, Nahrstedt A. Proanthocyanidins from Ginkgo biloba leaf extract and their radical scavenging activity. Pharm Biol 2011; 49: 471-476

[15] Symma N, Sendker ], Petereit F, Hensel A. Multistep analysis of diol-LCESI-HRMS data reveals proanthocyanidin composition of complex plant extracts (PAComics). J Agric Food Chem 2020; 68: 8040-8049 